\title{
Compliance with pharmacological treatment among patients after minimally invasive coronary bypass grafting
}

\author{
Łukasz J. Krzych ${ }^{1}$, Małgorzata Lach ${ }^{1}$, Sara Mustafa ${ }^{1}$, Michał Joniec ${ }^{1}$, \\ Marcelina Niemiec ${ }^{1}$, Maciej Wybraniec ${ }^{2}$, Marek Cisowski ${ }^{1}$, Andrzej Bochenek ${ }^{1}$ \\ ${ }^{1} 1^{\text {st }}$ Department of Cardiac Surgery, Medical University of Silesia, \\ University Hospital No. 7, Upper Silesia Medical Center, Katowice, Poland \\ ${ }^{2} 1^{\text {st }}$ Department of Cardiology, Medical University of Silesia, \\ University Hospital No. 7, Upper Silesia Medical Center, Katowice, Poland
}

\begin{abstract}
Background: We sought to evaluate patients' adherence to optimal pharmacotherapy as recommended by the European Society of Cardiology, together with the assessment of potential clinical determinants of medical non-compliance in a large cohort of patients after endoscopic atraumatic coronary artery bypassing (EACAB).

Methods: This cross sectional study was conducted in a group of 706 individuals who underwent EACAB between April 1998 and December 2010. Data covering current pharmacological treatment with antiplatelet agents, beta-blockers $(B B)$ (or heart rate lowering calcium channel blockers [CCB] in case of intolerance and/or poor efficacy of beta-blockade), angiotensin-converting enzyme (ACE) inhibitors (or angiotensin receptor blockers [ARB]) and statins was acquired. Mean duration of observation after the surgery was $2132 \pm 1313$ days.

Results: Complete follow-up data has been obtained from 415 living patients (341 males). Amongst them, 353 (85\%) received antiplatelet agents, while BB or CCB were routinely ingested by 349 (84\%) patients. Statins were used by 310 (74.7\%) individuals and 274 (66\%) subjects took ACE inhibitors or ARB. Baseline demographic and clinical features, including major co-morbidities had no impact on patients' compliance with all investigated medications. There was no clear association between adherence to treatment and risk of rehospitalization or occurrence of major cerebral and cardiovascular events.

Conclusions: EACAB patients' compliance with pharmacotherapy guidelines is insufficient and is unrelated to demographic and clinical features of the subjects. Multidisciplinary approach involving health education, enhancement in prescription drug affordability and a better rapport between doctors and patients should be incorporated into clinical practice to overcome therapeutic disobedience. (Cardiol J 2013; 20, 6: 648-654)
\end{abstract}

Key words: cardiovascular, cardiac surgery, drug therapy, secondary prevention

Address for correspondence: Łukasz Krzych, MD, PhD, Associate Professor, $1^{\text {st }}$ Department of Cardiac Surgery, Medical University of Silesia, ul. Ziołowa 47, 40-635 Katowice, Poland, tel: +48 3235986 11, fax: +48 322527066 , e-mail: 1.krzych@wp.pl

Received: 06.02.2013 Accepted: 10.04.2013 


\section{Introduction}

Coronary surgery remains a gold standard of treatment for a vast majority of patients with proximal left anterior descending artery stenosis [1]. Minimally-invasive totally endoscopic atraumatic coronary artery bypassing (EACAB) represents a unique off-pump procedure aimed at minimizing surgical trauma. By design, this approach eventually introduced an era of patient-oriented cardiac surgery associated with lesser degree of postoperative pain, early implementation of rehabilitation, shortened in-hospital stay and improved quality of life in contrast to on-pump techniques requiring median sternotomy [2].

Despite overt benefits for patients, EACAB constitutes an ultimately challenging surgical technique with a steep learning curve. Since methods of surgical revascularization are reaching an immensely complex level, a question of patients' adherence to concurrent therapy rises. Apart from lifestyle modification, pharmacological treatment remains a cornerstone of secondary prevention of coronary artery disease (CAD) [3]. Primary and secondary prevention is of utmost importance for the reduction of cardiovascular morbidity and mortality [4]. Lifelong administration of antiplatelet agents, beta-blockers (BB), angiotensin-converting enzyme (ACE) inhibitors or angiotensin receptor antagonists and statins is thus strongly recommended by the European Society of Cardiology (ESC) guidelines [5]. Still, only half of the patients suffering from chronic diseases in developed countries follow treatment recommendations [6].

High satisfaction rate reported in patients after EACAB [7] makes them, in theory, perfect candidates for the evaluation of individual determinants of pharmacological compliance, as opposed to patients undergoing standard on-pump coronary artery bypass grafting (CABG) fraught with local and systemic complications. Previous reports have extensively dealt with socioeconomic and educational aspects of drug non-compliance [8-10], however, clinical characteristics of pharmacologically non-adherent individuals still remain vague. More to the point, no convincing data exists concerning secondary medical prevention in subjects after EACAB. Thus, the authors intended to conduct a diligent evaluation of patients' adherence to optimal pharmacotherapy as established by the ESC guidelines, together with the assessment of potential clinical risk factors of medical non-compliance in a large cohort of cardiac surgery patients.

\section{Methods}

We performed an epidemiological, cross-sectional study. The study group comprised 706 consecutive patients with single-vessel (76.5\%) or multi-vessel (23.5\%) CAD, who underwent EACAB between April 1998 and December 2010. Baseline demographic and clinical characteristics of the patients, including the presence of concomitant diseases, were obtained from hospital records. Charlson comorbidity index [11] was calculated. Post-operative pharmacological treatment was also assessed. All patients gave their written informed consent to participate in the study. The study was approved by local Ethics Committee and complies with the Declaration of Helsinki.

The follow-up investigation was performed between February and December 2011. Mean duration of follow-up was $2132 \pm 1313$ (median 1918.5, longest 4661) days. Data covering current pharmacological treatment with antiplatelet agents, BB (or heart rate lowering calcium channel blockers $[\mathrm{CCB}]$ in case of intolerance and/or poor efficacy of beta-blockade), ACE inhibitors or angiotensin receptor blockers (ARB) and statins was acquired by means of structured telephone interview. In case of no response or unknown phone number, a standardized questionnaire was sent by post. All patients were subsequently interviewed with respect to rehospitalization and the occurrence of major cardiac and cerebrovascular events (MACCE), which were defined as: myocardial infarction (MI), repeat revascularization, and stroke or transient ischemic attack (TIA). The number of deceased patients was verified with national registry of cardiac surgery procedures.

Impact of baseline demographic and clinical features on adherence to secondary pharmacological prevention was assessed. Additionally, we evaluated patients' prognosis with relation to their compliance with the recommended treatment.

\section{Statistical analysis}

Statistical analysis was performed using the MedCalc software (v11.0.1). Data is shown as mean and standard deviation for quantitative variables, and as relative values and percentage for qualitative variables. In-group differences in quantitative variables were tested using analysis of variance (for normally distributed variables) or the Kruskal-Wallis test (for non-normally distributed variables). Normality of the data distribution was 
Table 1. Baseline characteristics of the subjects.

\begin{tabular}{lc}
\hline Age [years] & $59 \pm 6$ \\
Male gender & $341(82.2 \%)$ \\
Body mass index (BMI) $\left[\mathrm{kg} / \mathrm{m}^{2}\right]$ & $27.7 \pm 3.8$ \\
$\mathrm{BMI} \geq 25 \mathrm{~kg} / \mathrm{m}^{2}$ & $317(76.4 \%)$ \\
Charlson comorbidity index & $3 \pm 1$ \\
[points] & \\
Diabetes mellitus & $73(17.6 \%)$ \\
Previous MI & $164(39.5 \%)$ \\
Previous PCl & $98(23.6 \%)$ \\
Arterial hypertension & $225(54.2 \%)$ \\
Peripheral artery disease & $8(1.9 \%)$ \\
COPD & $12(2.9 \%)$ \\
Chronic renal failure & $8(1.9 \%)$ \\
Smoking habit & $174(42 \%)$ \\
LVEF $[\%]$ & $54 \pm 7$ \\
\hline
\end{tabular}

$\mathrm{Ml}$ - myocardial infarction; $\mathrm{PCl}$ - percutaneous coronary intervention; COPD — chronic obstructive pulmonary disease LVEF - left ventricular ejection fraction

verified using the Shapiro-Wilk test. Chi-square test was used for qualitative variables. Odds ratios with $95 \%$ confidence intervals were calculated to evaluate basic determinants of patients' compliance and its association with outcome. $\mathrm{P}<0.05$ was considered statistically significant.

\section{Results}

\section{Patients' characteristics}

In a prospective observation 20 (2.8\%) patients died. Complete follow-up data has been obtained from 415 living patients, 74 females and 341 males (study response rate $58.8 \%$ ). Detailed patient characteristics are shown in Table 1 . In survivors, a total of $98(23.6 \%)$ cardiac and cerebrovascular events were noticed: 26 (6.3\%) had MI, 11 (2.6\%) underwent stroke or TIA and $61(14.7 \%)$ had repeat revascularization (only percutaneous coronary interventions [PCI]).

\section{Pharmacological treatment in a follow-up}

The majority of patients received drug therapy as recommended by the ESC [5]. 353 (85\%) patients received antiplatelet agents (including acetylsalicylic acid [ASA], clopidogrel or ticlopidine), while $\mathrm{BB}$ or $\mathrm{CCB}$ were routinely ingested by 349 (84\%) patients. Statins were used by 310 (74.7\%) of the EACAB patients. Only 274 (66\%) subjects took ACE inhibitors or ARB.

\section{ASA/clopidogrel/ticlopidine}

It was demonstrated that gender $(\mathrm{p}=0.9)$, diabetes mellitus $(p=0.9)$, hypertension $(\mathrm{p}=0.8)$, previous MI ( $\mathrm{p}=0.9)$ or PCI ( $=0.3)$, Charlson index $(\mathrm{p}=0.4)$ and ejection fraction $(\mathrm{EF})(\mathrm{p}=0.1)$ had no impact on ingestion of antiplatelet agents. Patients who did not use antiplatelet drugs were on average 5 years older compared to those who complied with treatment $(\mathrm{p}=0.007)$ (Table 2, Fig. $1 \mathrm{~A})$. But the mean time of follow-up of the patients who were treated with the agents was also shorter compared to patients not using these drugs (1400 days vs. 1900 days, respectively; $p=0.01$ ).

Patients who used antiplatelet agents less frequently underwent rehospitalization (of any cause) compared to those who did not comply with treatment $(49.7 \%$ vs. $66.1 \%$; $=0.02)$. In patients who received antiplatelet drugs MACCE were observed as frequently as in those who did not ( $18.8 \%$ vs. $20.4 \%$; $\mathrm{p}=0.9$ ). MI was unrelated to subjects' compliance with antiplatelet drugs (6.6\% vs. $4.8 \%$; $\mathrm{p}=0.8)$. Treatment with antiplatelet medications was positively associated with the fact of repeat revascularization ( $16.6 \%$ vs. $4.8 \%$; $\mathrm{p}=0.02$ ). Stroke occurrence was not related with treatment with antiplatelet drugs $(2.3 \%$ vs. $4.8 \%$; $\mathrm{p}=0.4)$ (Fig. 2A).

\section{Beta-blockers/calcium channel blockers}

Amongst baseline features, it was found that gender $(\mathrm{p}=0.9)$, diabetes mellitus $(\mathrm{p}=0.3)$, hypertension $(\mathrm{p}=0.8)$, previous $\mathrm{MI}(\mathrm{p}=0.8)$ or PCI

Table 2. Impact of preoperative qualitative demographic and clinical variables on patients' compliance in a follow-up.

\begin{tabular}{lcccc}
\hline Variable & Antiplatelet agent & BB/CCB & ACEI/ARB & Statin \\
\hline Male gender & $0.98(0.47-2.06)$ & $0.97(0.48-1.98)$ & $0.75(0.44-1.28)$ & $1.40(0.74-2.65)$ \\
Diabetes & $1.13(0.52-2.44)$ & $0.69(0.34-1.37)$ & $1.37(0.75-2.48)$ & $0.75(0.41-1.37)$ \\
Previous MI & $0.96(0.55-1.67)$ & $1.09(0.63-1.87)$ & $1.34(0.88-2.04)$ & $1.25(0.79-1.99)$ \\
Previous PCI & $1.52(0.78-2.96)$ & $0.77(0.43-1.38)$ & $0.96(0.6-1.56)$ & $1.79(1.03-3.13)^{*}$ \\
Arterial hypertension & $0.92(0.52-1.62)$ & $0.93(0.63-1.87)$ & $0.97(0.63-1.5)$ & $0.92(0.57-1.47)$ \\
\hline
\end{tabular}

Values are odds ratio with $95 \%$ confidence interval (in brackets), ${ }^{*} p<0.05$; ACE - angiotensin-converting enzyme inhibitor ARB - angiotensin receptor blockers; BB — beta-blocker; CCB — calcium channel blocker; other abbreviations as in Table 1 


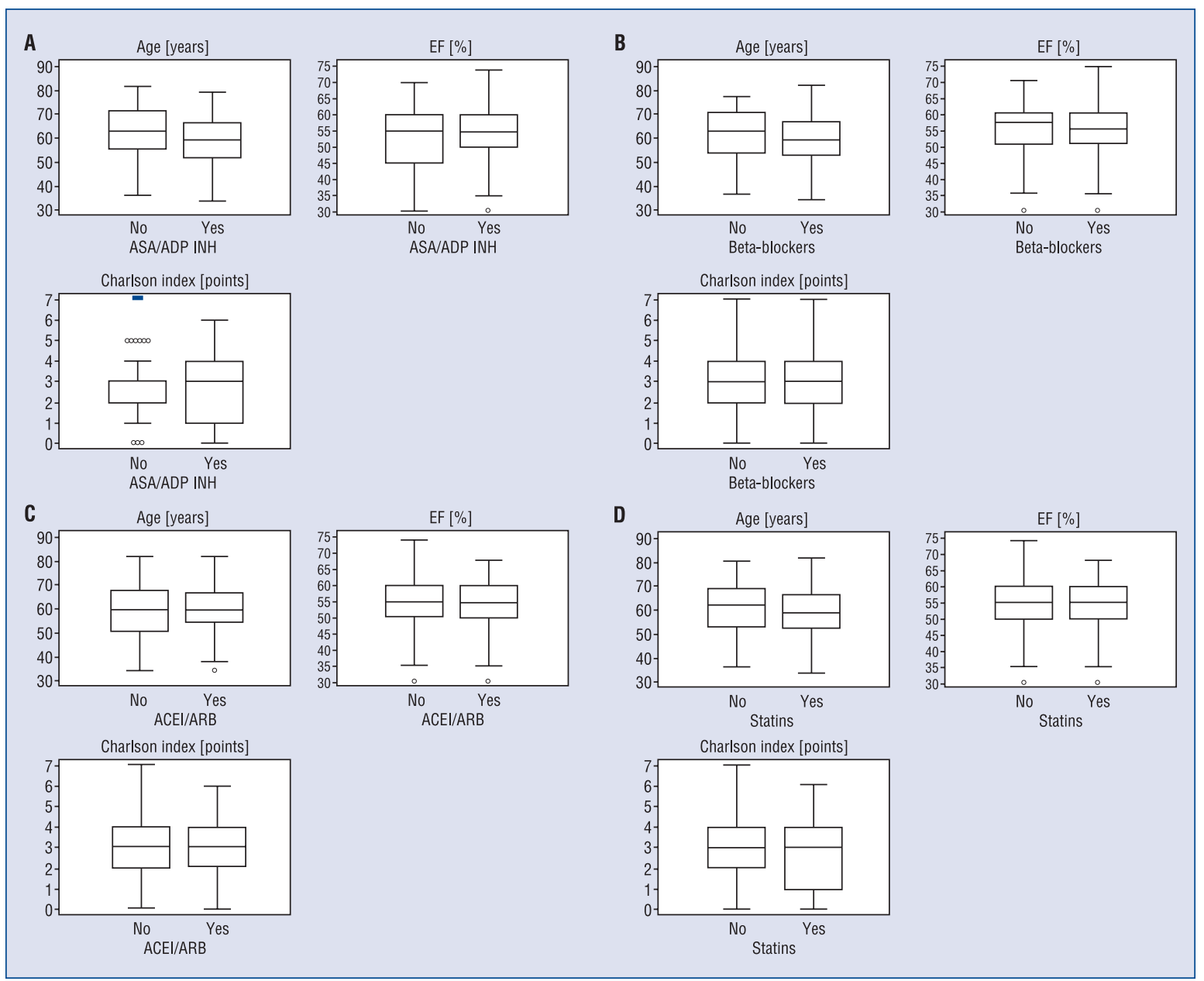

Figure 1. Impact of preoperative quantitative demographic and clinical variables on patients' compliance in a follow-up; A. Antiplatelet agents; B. Beta-blockers/calcium channel blocker; C. Angiotensin-converting enzyme inhibitors (ACEI)/angiotensin receptor blockers (ARB); D. Statins; ASA — acetylsalicylic acid; ADP INH — ADP-receptor inhibitors.

$(p=0.4)$, Charlson index $(p=0.7)$, age of subjects $(\mathrm{p}=0.06)$ and $\mathrm{EF}(\mathrm{p}=0.5)$ were not associated with treatment with BB or CCB (Table 2, Fig. 1B). The mean time of follow-up of the patients who were treated with the agents was slightly shorter compared to patients not using these drugs (1500 days vs. 1900 days, respectively; $\mathrm{p}<0.001$ ).

Patients who ingested BB/CCB were rehospitalized as frequently as those who were not taking medications (50.8\% vs. $59 \%$; $\mathrm{p}=0.3$ ). MACCE were observed as frequently in patients who received $\mathrm{BB}$ as in those who did not (19.8\% vs. $22.6 \%$; $\mathrm{p}=0.8$ ). MI was unrelated to subjects' compliance with $\mathrm{BB} / \mathrm{CCB}(7.0 \%$ in those who used vs. $3.0 \%$ in those who did not use; $\mathrm{p}=0.3$ ), as well as repeat revascularization ( $15.9 \%$ vs. $9.1 \%$; $p=0.2$ ). Stroke occurrence was not also associated with treatment with $\mathrm{BB} / \mathrm{CCB}(2.3 \%$ vs. $4.5 \%$; $\mathrm{p}=0.5)$ (Fig. $2 \mathrm{~B})$.

\section{ACE inhibitors/ARB}

None of the investigated preoperative variables correlated with treatment with ACE inhibitors: gender $(\mathrm{p}=0.3)$, diabetes mellitus $(\mathrm{p}=0.4)$, hypertension $(\mathrm{p}=0.9)$, previous $\mathrm{MI}(\mathrm{p}=0.2)$ or PCI $(p=0.7)$, Charlson index $(p=0.6)$, age $(p=0.7)$ and EF ( $\mathrm{p}=0.8$ ) (Table 2, Fig. 1C). The follow-up duration also did not influence taking ACE inhibitors/ARB in secondary prevention $(\mathrm{p}=0.2)$.

There was no association between the fact of receiving ACE inhibitors/ARB and the fact of rehospitalization ( $54 \%$ vs. $48.6 \%$; $\mathrm{p}=0.3$ ) or MACCE occurrence $(21.4 \%$ vs. $17.6 \% ; \mathrm{p}=0.4)$. MI was also unrelated to subjects' compliance with ACE inhibitors/ARB (7\% in those who used vs. $5 \%$ in those who did not use; $p=0.6$ ), as well as repeat revascularization $(16.5 \%$ vs. $11.4 \% ; \mathrm{p}=0.2)$ and stroke/TIA (2.2\% vs. $3.5 \%$; $\mathrm{p}=0.6$ ) (Fig. $2 \mathrm{C}$ ). 


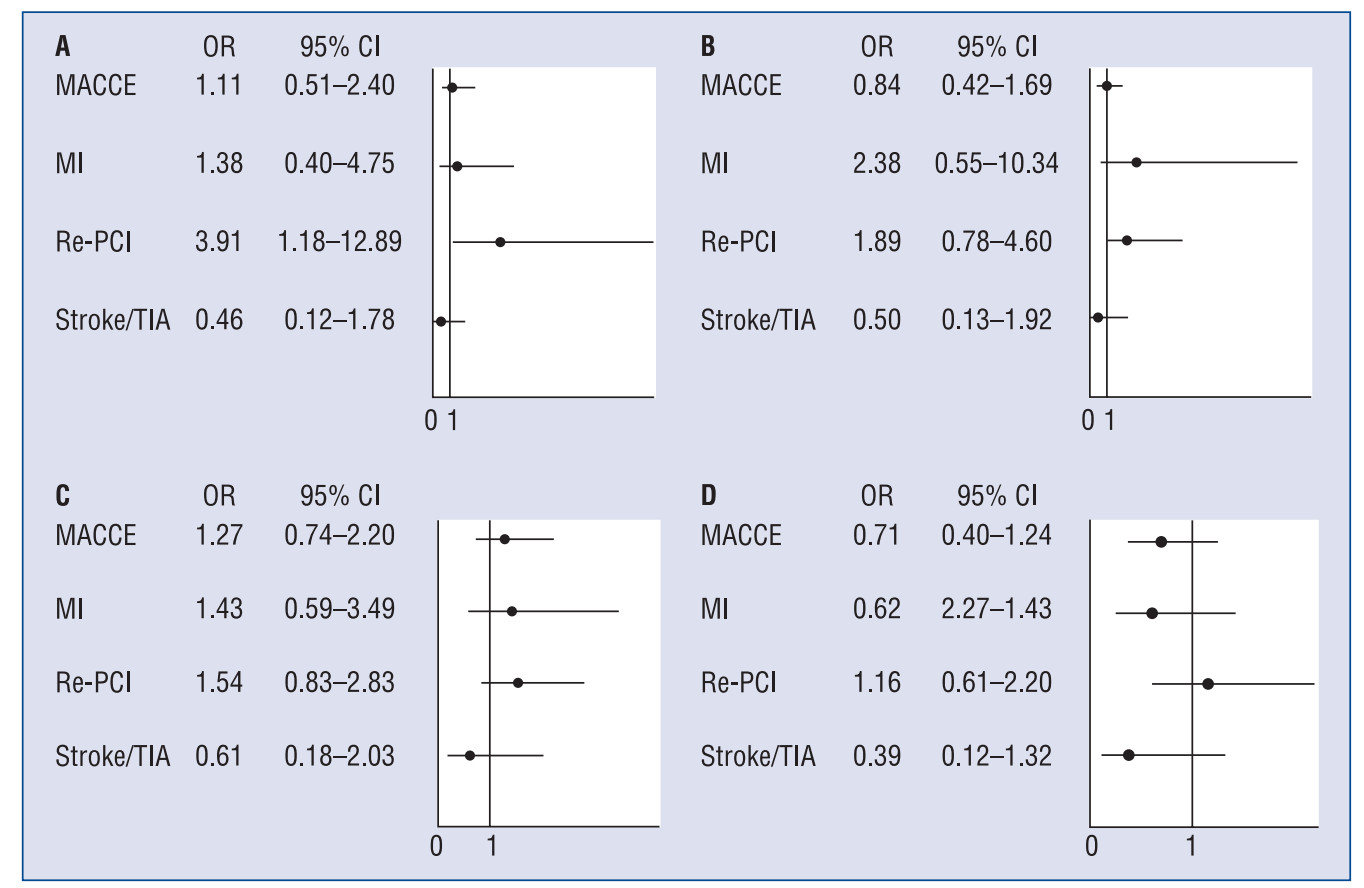

Figure 2. Association between pharmacotherapy and major cerebral and cardiovascular events (MACCE); A. Antiplatelet agents; B. Beta-blockers/calcium channel blocker; C. Angiotensin-converting enzyme inhibitors/angiotensin receptor blockers; D. Statins; $\mathrm{MI}$ - myocardial infarction; re- $\mathrm{PCl}$ - repeat revascularization with percutaneous coronary intervention; TIA — transient ischemic attack.

\section{Statins}

As far as baseline variables are concerned, gender $(p=0.4)$, diabetes $(p=0.4)$, hypertension $(\mathrm{p}=0.8)$, history of MI $(\mathrm{p}=0.4)$ and Charlson index $(\mathrm{p}=0.3)$ were unrelated with treatment with statins. Patients with a history of PCI received statins more frequently than those with no previous PCI $(p=0.03)$. Age of subject $(p=0.1)$, $\mathrm{EF}(\mathrm{p}=0.6)$ had no association with statins' ingestion (Table 2, Fig. 1D). The follow-up duration was statistically significantly related with patients' compliance with statins - it was shorter in those subjects who were taking statins (1570 days vs. 2016 days in those who did not receive statins; $\mathrm{p}<0.001)$.

Patients treated with statins less frequently underwent rehospitalization $(48.1 \%$ vs. $64.4 \%$; $\mathrm{p}=0.005)$. In patients who received statins, MACCE were documented with similar frequency compared to those who did not receive them $(18.9 \%$ vs. $24.7 \%$; $\mathrm{p}=0.3$ ). MI occurrence was not associated with patients' compliance $(5.5 \%$ vs. $8.6 \%$; $\mathrm{p}=0.4)$, as well as repeat revascularization ( $15.2 \%$ vs. $13.5 \% ; \mathrm{p}=0.8$ ) or stroke/TIA ( $2 \%$ vs. $5 \% ; \mathrm{p}=0.2$ ) (Fig. 2D).

\section{Discussion}

The present study has investigated the association between patients' compliance with recommended pharmacological treatment and baseline clinical variables, and postoperative cardiovascular events in a large cohort of patients after EACAB. This analysis appears to be crucial because of the paucity of similar data in literature. The knowledge concerning clinical characteristics of potentially non-compliant individuals might help improve ingestion of recommended medications by means of actions devoted particularly to those subjects.

The implementation of pharmacotherapy after cardiac surgery is of great importance as it reduces mortality, morbidity, risk of rehospitalization and increases quality of life. We found that fulfillment of recommended secondary prevention guidelines was insufficient in relation to all groups of drugs we took into account. Similar results were obtained by several investigators [12-14]. In the retrospective study of Vermeer and Bajorek [12] in patients 3 months after MI only $82 \%$ subjects used BB, $86 \%$ had statin therapy and $79 \%$ received either $\mathrm{ACE}$ inhibitor or $\mathrm{ARB}$. Worrisome results are given 
by Lee et al. [13] who underlined unsatisfactory compliance with pharmacological treatment 3 months after acute coronary syndrome. Their study revealed that only $63.9 \%$ of patients were receiving $\mathrm{BB}, 62.6 \%$ ingested statins and $51.8 \%$ had ACE inhibitor/ARB therapy [13]. Moreover, Griffo et al. [14] revealed that 12 months after percutaneous revascularization $94 \%$ of individuals were taking antiplatelet agents, $87 \%$ statins, $80.7 \%$ $\mathrm{BB}$ and $81.1 \%$ received ACE inhibitors. Regardless of statistical variations, all the above-mentioned reports stay consistent and disconcerting.

In the light of current ESC recommendations $[3,5]$ underpinning the importance of 4-drug regimen in stable patients after acute coronary syndrome, the observed discrepancy between guidelines and real medical practice substantiates further investigation into the risk factors of non-compliance. One of them is inappropriate implementation of the guidelines by physicians who are responsible for postoperative pharmacotherapy. This problem was described by Fox et al. [15] who documented that only $92 \%$ of CABG patients were ordered ASA, $70 \% \mathrm{BB}, 73 \%$ had statins and $26 \%$ were prescribed ACE inhibitors at discharge. In a similar study it was shown that $92 \%$ of patients were recommended to take ASA, $79 \% \mathrm{BB}$, $55 \%$ ACE inhibitor or ARB and 92\% statins [16]. Additionally, this important gap between current guidelines on cardiovascular pharmacotherapy and every-day clinical practice was illustrated in terms of primary prevention in Spanish primary health care units [17].

Secondly, contraindications and side effects account only for small portion of drug cessation. They include hypotension after ACE inhibitors or $\mathrm{ARB}$, bradycardia following $\mathrm{BB}$ administration, major bleeding caused by antiplatelet agents and myopathy after statins. Yet, none of the respondents demonstrated any of the abovementioned symptoms.

Dosing time of certain pharmacologic agents is, beyond doubt, a key factor contributing to non-compliance. Statins, ACE inhibitors and ARB are frequently ingested before sleep and therefore patients tend to withdraw or forget their nocturnal medications, whereas the introduction of once-daily formulations has proven beneficial in terms of adherence to evidence-based therapies [10]. Furthermore, there are no fixed combinations on the market combining any of the 4 drug regimen analyzed in our study. It has been well documented that high number of pills corresponds with poor compliance. Frequent termination of crucial drugs revealed by our study may be partially triggered by financial limitations, especially among seniors, due to high prices of drugs for the chronically ill patients or lack of social support. Lack of continuity of pharmacotherapy due to poor relay of clinical data between specialists and general practitioners often results in alteration of therapy, since discharge summaries are not directly mailed to outpatient clinic in the setting of Polish healthcare system.

Patients' self-confidence of being cured by a complex cardiac procedure, endorsed by rapid improvement of physical exertion tolerance, may also be an incentive to cessation of pharmacotherapy or even failure to fill the prescription [18].

Last but not least, a potent demographic feature in the form of education cannot be neglected as it directly affects the level of awareness and commitment to overcome the disease. The idea of patients' involvement in the process of therapy derives from the salutogenic model of health proposed by Antonovsky, which pursues psychosocial determinants of medical condition [19]. Lifestyle modification is regarded as the most efficient way of dealing with modifiable cardiovascular risk factors. In line with these principles of health promotion, therapeutic non-adherence is widely recognized as a vital, yet often underestimated cardiovascular risk factor common for all populations [20].

Interestingly, we have revealed some association between compliance and MACCE occurrence. The patients who experienced repeat revascularization with PCI more frequently used antiplatelet drugs, which can probably be attributed to more careful approach of attending physicians alert to severe complications, such as stent thrombosis. It is in line with current recommendation as the treatment reduces risk of stent occlusion and subsequent risk of all cardiovascular events, including death which was confirmed in several studies [21]. Also, we found a correlation between ingestion of antiplatelet agents and $\mathrm{BB}$, and the need for readmission. Compliant individual less frequently experienced hospitalization compared to patients indifferent to pharmacotherapy, hence we assume that pharmacological interventions help improve patients' outcome and reduce costs for the healthcare system [21-26]. Although our study failed to demonstrate a statistically significant link between patients' clinical features and drug compliance, we managed to denote considerable prevalence of the issue of therapeutic non-adherence and its clinical implications. 


\section{Limitations of the study}

Unfortunately this study has several drawbacks that should be taken into account in data interpretation. Firstly, although with prospective follow-up, the study is cross-sectional in its design. Accordingly, we may not assess cause - effect relationship between medications which have been taken and the incidence of MACCE. As a result, we also did not adjudicate the influence of the follow-up duration on the results. Nonetheless, we performed a sub-analysis of impact of follow-up time on the subjects' amenability in which we tried to overcome this limitation to some extent. Secondly, as we interviewed about $60 \%$ of the cohort, this research may generate selection bias, as well as recall bias in remaining participants. Finally, we did not attempt at minimizing the effect of confounders as we hadn't performed a multivariate analysis. However, we found only minor determinants of patients' compliance so the implementation of regression model was rather impractical.

\section{Conclusions}

EACAB patients' compliance with pharmacotherapy guidelines is rather insufficient with regard to current ESC recommendations. We conclude that drug non-adherence does not correspond with demographic and clinical features of the subjects. For improvement, multidisciplinary approach including patients' education, enhancement in therapeutic rapport between doctors and patients, as well as improvement of healthcare system should be incorporated into clinical practice.

\section{Acknowledgements}

This study was supported by the Polish Ministry of Science and Higher Education grant No. NN-6-342/07.

\section{Conflict of interest: none declared}

\section{References}

1. Guidelines on myocardial revascularization, The Task Force on Myocardial Revascularization of the European Society of Cardiology (ESC) and the European Association for Cardio-Thoracic Surgery (EACTS). Eur Heart J, 2010; 31: 2501-2555.

2. Jegaden O, Wautot F, Sassard T et al. Is there an optimal minimally invasive technique for left anterior descending coronary artery bypass? J Cardiothorac Surg, 2011; 6: 37.

3. European Guidelines on cardiovascular disease prevention in clinical practice (version 2012). Eur J Prev Cardiol, 2012; 19: 585-667.

4. Joseph P, Teo K. Optimal medical therapy, lifestyle intervention, and secondary prevention strategies for cardiovascular event reduction in ischemic heart disease. Curr Cardiol Rep, 2011; 13: 287-295
5. Fox K, Garcia MA, Ardissino D et al. Task Force on the Management of Stable Angina Pectoris of the European Society of Cardiology; ESC Committee for Practice Guidelines (CPG): Guidelines on the management of stable angina pectoris: executive summary: the Task Force on the Management of Stable Angina Pectoris of the European Society of Cardiology. Eur Heart J, 2006; 27: 1341-1381.

6. World Health Organization. Adherence to Long-Term Therapies: Evidence for Action. World Health Organisation, Geneva, 2003 (Web access: http://www.who.int/chp/knowledge/publications/ /adherence full report.pdf).

7. Semere $\mathrm{WG}, \mathrm{E}$ dwards TM, Boyd D et al. The world wide web and robotic heart surgery. Heart Surg Forum, 2003; 6: E111-E119.

8. Miller NH. Adherence behavior in the prevention and treatment of cardiovascular disease. J Cardiopulm Rehabil Prev, 2012; 32: $63-70$.

9. German PS. Compliance and chronic disease. Hypertension, 1988; 11(3 Part 2): II56-II60

10. Frishman WH. Importance of medication adherence in cardiovascular disease and the value of once-daily treatment regimens. Cardiol Rev, 2007; 15: 257-263.

11. Charlson ME, Pompei P, Ales KL, MacKenzie CR. A new method of classifying prognostic comorbidity in longitudinal studies: Development and validation. J Chron Dis, 1987; 40: 373-383.

12. Vermeer NS, Bajorek BV. Utilization of evidence-based therapy for the secondary prevention of acute coronary syndromes in Australian practice. J Clin Pharm Ther, 2008; 33: 591-601.

13. Lee HY, Cooke CE, Robertson TA. Use of secondary prevention drug therapy in patients with acute coronary syndrome after hospital discharge. J Manag Care Pharm, 2008; 14: 271-280.

14. Griffo R, Ambrosetti M, Tramarin R et al. Effective secondary prevention through cardiac rehabilitation after coronary revascularization and predictors of poor adherence to lifestyle modification and medication. Results of the ICAROS Survey. Int J Cardiol, 2013; 167: 1390-1395.

15. Fox DJ, Kibiro M, Eichhöfer J, Curzen NP. Patients undergoing coronary revascularisation: A missed opportunity for secondary prevention? Postgrad Med J, 2005; 81: 401-403.

16. Turley AJ, Roberts AP, Morley R. Secondary prevention following coronary artery bypass grafting has improved but remains sub-optimal: the need for targeted follow-up. Interact Cardiovasc Thorac Surg, 2008; 7: 231-234.

17. Lopez-Carmona D, Bernal-Lopez M, Mancera-Romero J et al. Compliance with cardiovascular drug prevention measures in a general population: The Multidisciplinary Intervention in Primary Care (IMAP) study. Eur J Cardiovasc Prev Rehabil, 2011; 19: 1074-1081.

18. Miller NH. Compliance with treatment regimens in chronic asymptomatic diseases. Am J Med, 1997; 102: 43-49.

19. Eriksson M, Lindström B. A salutogenic interpretation of the Ottawa Charter. Health Promot Int, 2008; 23: 190-199.

20. Munger MA, Van Tassell BW, LaFleur J. Medication nonadherence: An unrecognized cardiovascular risk factor. Med Gen Med, 2007; 9: 58.

21. Dai Y, Ge J. Clinical use of aspirin in treatment and prevention of cardiovascular disease. Thrombosis, 2012; 2012: 245037.

22. Kubota N, Kasai T, Miyauchi K et al. Therapy with statins and aspirin enhances long-term outcome of percutaneous coronary intervention. Heart Vessels, 2008; 23: 35-39.

23. Rockson SG, deGoma EM, Fonarow GC. Reinforcing a continuum of care: In-hospital initiation of long-term secondary prevention following acute coronary syndromes. Cardiovasc Drugs Ther, 2007; 21 : 375-388

24. Kalavrouziotis D, Buth KJ, Cox JL, Baskett RJ. Should all patients be treated with an angiotensin-converting enzyme inhibitor after coronary artery bypass graft surgery? The impact of angiotensin-converting enzyme inhibitors, statins, and beta-blockers after coronary artery bypass graft surgery. Am Heart J, 2011; 162: 836-843

25. Kulik A, Brookhart MA, Levin R, Ruel M, Solomon DH, Choudhry NK. Impact of statin use on outcomes after coronary artery bypass graft surgery. Circulation, 2008; 118: 1785-1792.

26. Zhang Z, Marroquin OC, Weissfeld JL et al. Beneficial effects of statins after percutaneous coronary intervention. Eur J Cardiovasc Prev Rehabil, 2009; 16: 445-450. 